\title{
Influencia del tipo de sinterizado en el comportamiento anticorrosivo de recubrimientos sol-gel
}

Jorge Hernando Bautista-Ruiz ${ }^{1 *} \mid$ César Ortiz ${ }^{1} \mid$ Enrique Vera $^{1} \mid$

Recibido:

18 de agosto de 2008

Aceptado:

20 de noviembre de 2008

\section{Resumen}

Este trabajo muestra un estudio electroquímico de recubrimientos $\mathrm{SiO}_{2}$ $\mathrm{TiO}_{2} \mathrm{ZrO}_{2}$ obtenidos por el método sol-gel, sinterizados vía plasma y vía convencional. Los recubrimientos se conformaron a partir de $\mathrm{Si}\left(\mathrm{OC}_{2} \mathrm{H}_{5}\right)_{4}$, $\mathrm{Ti}(\mathrm{OBu})_{4}$ y $\mathrm{Zr}\left(\mathrm{OC}_{3} \mathrm{H}_{7}\right)_{4}$, se depositaron sobre sustratos de acero ANSI/304 mediante dip-coating en monocapa y bicapa. El proceso de densificado vía plasma se realizó a temperaturas del sustrato de $250{ }^{\circ} \mathrm{C}, 300{ }^{\circ} \mathrm{C}$ y $330{ }^{\circ} \mathrm{C}$ y el convencional a $400{ }^{\circ} \mathrm{C}$ y a una velocidad de calentamiento de $2{ }^{\circ} \mathrm{C} / \mathrm{min}$. El comportamiento anticorrosivo se estudió mediante las técnicas de espectroscopía de impedancia electroquímica (EIS) y Tafel. Se observó que el proceso de sinterización vía plasma, mejora la resistencia a la corrosión con respecto al método convencional.

Palabras clave: sol-gel, corrosión, plasma, EIS, Tafel.

\section{Abstract}

This work shows a comparative electrochemical study among the anticorrosive properties of coatings $\mathrm{SiO}_{2}, \mathrm{TiO}_{2}, \mathrm{ZrO}_{2}$ obtained by the method sol-gel and plasma sintering process and conventional. The coatings conformed to starting from $\mathrm{Si}\left(\mathrm{OC}_{2} \mathrm{H}_{5}\right)_{4}, \mathrm{Ti}(\mathrm{OBu})_{4}$ and $\mathrm{Zr}\left(\mathrm{OC}_{3} \mathrm{H}_{7}\right)_{4}$. These were deposited on steel substrate 304 by means of dip-coating in mono-layer and bi-layer. The plasma sintering process was carried out to temperatures of the substrate of $250{ }^{\circ} \mathrm{C}$,

Diciembre 2008 ISSN 0122-820X $300{ }^{\circ} \mathrm{C}$ and $330^{\circ} \mathrm{C}$ and the conventional to $400{ }^{\circ} \mathrm{C}$ and a speed of heating of $2{ }^{\circ} \mathrm{C} / \mathrm{min}$. the anticorrosive behavior was studied by means of the techniques of electrochemical impedance spectroscopy (EIS) and Tafel. It was observed that

1. GRUPO DE SUPERFICIES, ELECTROQUÍMICA Y CORROSIÓN -GSEC-. Escuela de Física, Facultad de Ciencias. Universidad Pedagógica y Tecnológica de Colombia, Av. Central del Norte, Tunja, Boyacá, Colombia. Tel: 57 (8) 7422360. the plasma sintering process, it improves the resistance to the corrosive attack to in regard to the conventional method.

Keywords: sol-gel, corrosion, plasma, EIS, Tafel 


\section{Introducción}

E 1 procedimiento sol-gel es uno de los métodos de síntesis de materiales que presenta gran interés en la actualidad $[1,2,3]$. Este procedimiento consta de tres etapas: la preparación del sol, la gelación del mismo y la eliminación del solvente [1]. El sol puede producirse a partir de una sal inorgánica o un precursor molecular, esencialmente metal-alcóxidos. En el seno del sistema se forma una red molecular debido a reacciones de condensación de especies hidrolizadas, y la microestructura que se conforma depende fuertemente de las condiciones experimentales. La hidrólisis del precursor molecular, su reacción con el agua, usualmente se realiza en presencia de un catalizador ácido o básico el cual permite un control de la velocidad y extensión de la reacción de hidrólisis. Diferentes ácidos han sido usados: $\mathrm{HCl}, \mathrm{HNO}_{3}$ o $\mathrm{CH}_{3} \mathrm{COOH}$. El mayor problema que se presenta, en la obtención de cerámicos multicomponentes, es la conformación de un gel homogéneo debido a que las velocidades de hidrólisis y condensación de los metalalcóxidos correspondientes a los cationes de interés pueden ser diferentes.

El sistema estudiado en este trabajo es el $\mathrm{SiO}_{2}-\mathrm{TiO}_{2}-\mathrm{ZrO}_{2}$, aplicado, sobre sustratos de acero AISI/304, en monocapa y bicapa [4]. Con este sistema es posible la elaboración de recubrimientos cerámicos que presenten baja expansión térmica, alto índice de refracción [5], y también como recubrimientos antirreflectivos o anticorrosivos [6].

Enla evaluación de propiedadesdieléctricas, de los recubrimientos, se utiliza la técnica EIS (Espectroscopiadeimpedanciaelectroquímica) $[7,8,9,10,11]$. Adicionalmente, se evalúan las propiedades anticorrosivas de las películas mediante diagramas de Tafel

\section{Desarrollo experimental}

Para la elaboración del sistema $\mathrm{SiO}_{2} \mathrm{TiO}_{2} \mathrm{ZrO}_{2}$ (sol) se utilizan como precursores Tetraethyl ortosilacate TEOS $\mathrm{Si}\left(\mathrm{OC}_{2} \mathrm{H}_{5}\right)_{4}$, Zirconium (IV) butoxide, $\mathrm{Zr}\left(\mathrm{OC}_{3} \mathrm{H}_{7}\right)_{4}$ y Titanium (IV) butoxide, $\mathrm{Ti}(\mathrm{OBu})_{4}$. Como solvente etanol, $\mathrm{EtOH}$ y como acomplejante ACAC. La figura 1, muestra el proceso desarrollado para la conformación del sol estable.

El sol del sistema $\mathrm{SiO}_{2} \mathrm{TiO}_{2} \mathrm{ZrO}_{2}$, se aplicó sobre chapas de acero AISI/304 de dimensiones $3,5 \mathrm{~cm} \times 2,50 \mathrm{~cm} \times 0,32 \mathrm{~cm}$, pulidas a brillo metalográfico, desengrasadas a ultrasonido con acetona y secadas con aire caliente. El paso siguiente fue conformar las películas mediante la técnica "Dip coating", utilizando una velocidad de extracción de 3,67 $\mathrm{cm} / \mathrm{min}$ previa elaboración del sol estable, en una concentración de los precursores $\mathrm{Si} / \mathrm{Ti} / \mathrm{Zr}$ : 10/70/20. El tiempo de envejecimiento del sol fue de 320 horas.

Figura 1. Proceso para la preparación del sol estable.

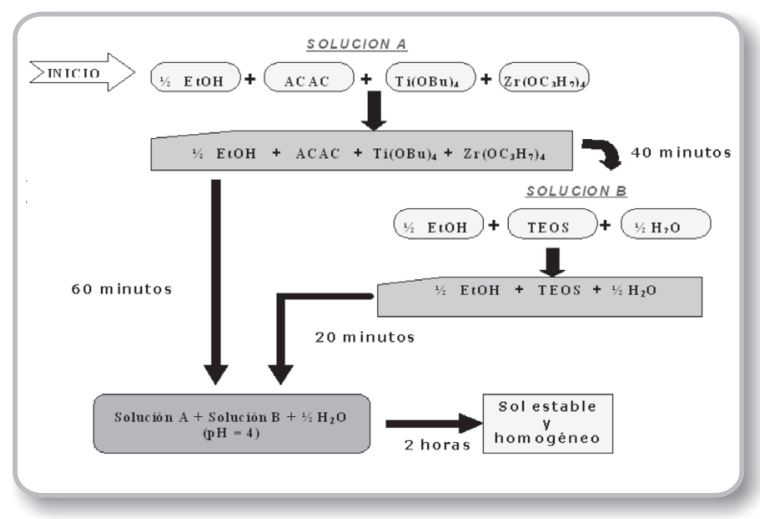

La sinterización convencional de las películas se realizó a una velocidad de calentamiento de $2{ }^{\circ} \mathrm{C} / \mathrm{min}$. Para la monocapa, durante una hora se mantiene la temperatura constante en $300{ }^{\circ} \mathrm{C}$, luego se aumenta a $400{ }^{\circ} \mathrm{C}$ por media hora. Para la bicapa, el tratamiento térmico fue a $100^{\circ} \mathrm{C}$ durante una hora, posteriormente a $200^{\circ} \mathrm{C}$ para un tiempo de media hora.

En la sinterización vía plasma, los sustratos de acero recubiertos en monocapa y bicapa son ubicados sobre el ánodo del reactor el cual se lleva a un vacío de 0,2 Torr. Entre los electrodos se aplica una diferencia de 
potencial de $600 \mathrm{~V}$ generando una descarga luminiscente DG (glow discharge) estable. Posteriormente se ajusta la variación del flujo de aire hasta obtener la temperatura de 330 ${ }^{\circ} \mathrm{C}$, censada en el volumen del sustrato. Este tratamiento se mantiene durante 2 horas.

Para los estudios de EIS se utilizó un equipo Gamry Instruments; este dispositivo consta de tres electrodos, un electrodo de referencia $(\mathrm{Ag} / \mathrm{AgCl})$, un contraelectrodo de platino y un electrodo de trabajo que es la pieza a ensayar. La frecuencia de barrido de la prueba, comprende un rango entre $0,01 \mathrm{~Hz}$ y $10^{5} \mathrm{~Hz}$. La amplitud de la señal sinusoidal utilizada es de $10 \mathrm{mV}$, en un área de exposición de 0,5 $\mathrm{cm}^{2}$ utilizando como solución de trabajo $\mathrm{HCl}$ al $3 \%$ con $\mathrm{pH}$ de 2,83. Los diagramas de Tafel se obtuvieron en un rango de $-0,2 \mathrm{~V}$ a $0,2 \mathrm{~V}$ con un barrido de $0,5 \mathrm{mV} / \mathrm{s}$. Estas condiciones de estudio se mantuvieron tanto para las películas sinterizadas por vía plasma como convencional.

\section{Resultados y discusión}

\section{Espectroscopia de impedancia electroquímica (EIS)}

Esencialmente los análisis EIS se centran en determinar los valores de resistencia a la polarización $(R p)$, la cual representa la oposición al paso de los iones de la solución de trabajo al sustrato, impidiendo que las probetas de acero se corroan. En general, cuando un recubrimiento registra un alto valor de $R p$ es posible concluir que es un buen protector anticorrosivo.

En la figura 2, se muestran los diagramas Bode para los recubrimientos sinterizados vía plasma y convencional. Se observa la correlación de la información obtenida mediante EIS de la impedancia y la frecuencia, para el sustrato AISI/304 y los recubrimientos en monocapa y bicapa. Se evidencia que la resistencia a la polarización $R p$ crece a medida que aumenta el número de capas, obteniéndose un mayor valor para la bicapa. Este aumento en el valor de la resistencia a la polarización $R p$ se correlaciona con la susceptibilidad que presentan los recubrimientos frente al proceso de corrosión [10]. Es de resaltar que al aumentar el número de capas cerámicas depositadas sobre el sustrato se optimiza la acción anticorrosiva, siendo la solución de $\mathrm{HCl}$ al $3 \%$ un agente muy agresivo. El mejor comportamiento anticorrosivo está ligado a una buena sinterización del recubrimiento. Adicionalmente, se aprecia que la resistencia de la solución de trabajo (Rs) tiene un valor promedio de $12,74 \Omega$.

Figura 2. Diagramas de Bode, (a) sinterizado vía plasma y (b) sinterizado convencional.

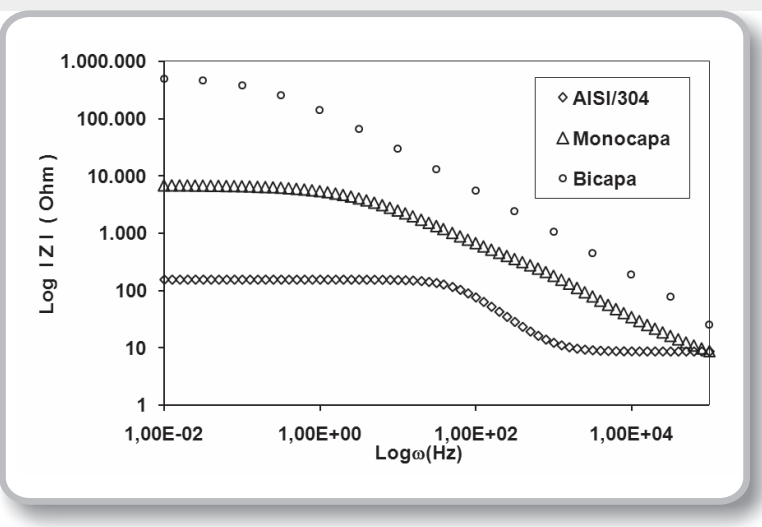

(a)

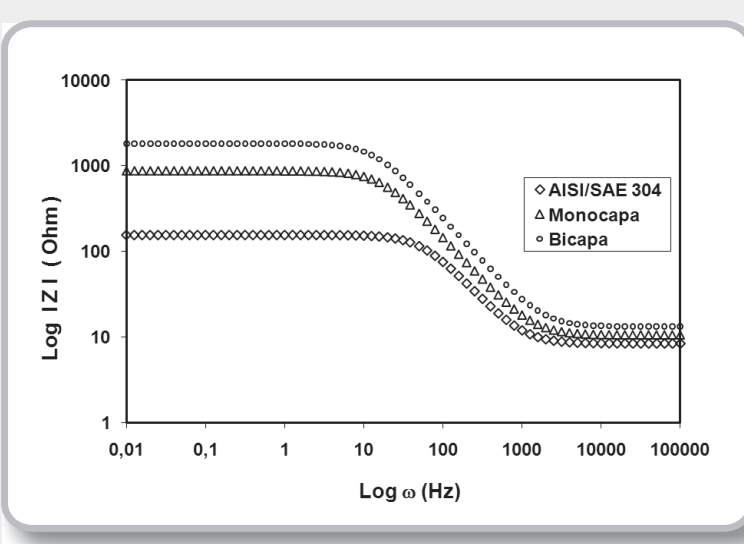

(b)
Diciembre 2008 ISSN 0122-820X 
Tabla 1. Valores de Rp para el sustrato y los recubrimientos sinterizados vía plasma y convencional.

\begin{tabular}{|c|c|c|c|}
\hline Vía de & \multicolumn{3}{|c|}{$\boldsymbol{R}_{p}(\mathrm{~K} \Omega)$} \\
\cline { 2 - 3 } sinterizado & Monocapa & Bicapa & AISI/304 \\
\hline Plasma & 2,86 & 136,10 & \multirow{2}{*}{0,15} \\
\hline Convencional & 0,87 & 1,80 & \\
\hline
\end{tabular}

La tabla 1 , registra los valores de $R p$ tanto del sustrato como de los recubrimientos cerámicos aplicados en monocapa y bicapa, obtenidas por los procesos de sinterización vía plasma y convencional. Estos valores son obtenidos de los diagramas de Bode de la figuras 2. Los resultados de $R p$ muestran una tendencia a aumentar en relación con el número de capas aplicadas sobre el sustrato. El mejor desempeño anticorrosivo se presenta en la bicapa sinterizada vía plasma, aunque el comportamiento frente a la corrosión de la monocapa es considerable con respecto a la repuesta a la corrosión del sustrato AISI/304. En general el aumento de la resistencia a la polarización $R p$ es directamente proporcional a número de capas aplicadas y a la temperatura a la cual se realiza el tratamiento de sinterizado. El proceso de sinterización vía convencional también aporta un aumento en la resistencia a la polarización si se compara con el sustrato. Según el estudio, la sinterización vía plasma permite en bicapa obtener los mejores resultados en cuanto a resistencia a la polarización.

\section{Curvas de Tafel}

En la figura 3, se presentan las gráficas de los diagramas de Tafel para el sustrato AISI/304 y los recubrimientos en monocapa y bicapa sinterizadas vía plasma y vía convencional. De ésta se obtieneinformación referenteal potencial, la corriente y la velocidad de corrosión, cuando los recubrimientos se encuentran inmersos en la solución de $\mathrm{HCl}$ al 3\%.

En general, el potencial de corrosión de los recubrimientos tiende a tomar valores más positivos, si se compara con el potencial del sustrato. Este comportamiento es similar en cuanto al número de películas obtenidas por el proceso de sinterización vía convencional. Esto indica que el sistema $\mathrm{Si} / \mathrm{Ti} / \mathrm{Zr}$ protege frente a fenómenos de corrosión, corroborando de esta manera lo obtenido en los análisis de EIS. Es de resaltar, en el proceso de sinterizados vía plasma, el buen desempeño anticorrosivo de los recubrimientos en bicapa con respecto a los recubrimientos en monocapa. Esto se coteja con los valores altos de resistencia a la polarización que se obtuvieron en los análisis EIS. Para los recubrimientos obtenidos por sinterizado vía convencional, se observa efectividad anticorrosiva de las películas según el número de capas aplicadas, pero comparando los dos procesos de sinterizado, el vía plasma arroja los mejores resultados.

Figura 3. Diagramas de Tafel para el sustrato AISI/304 y los recubrimientos en monocapa y bicapa (a) sinterizados vía plasma y (b) sinterizados por vía convencional.

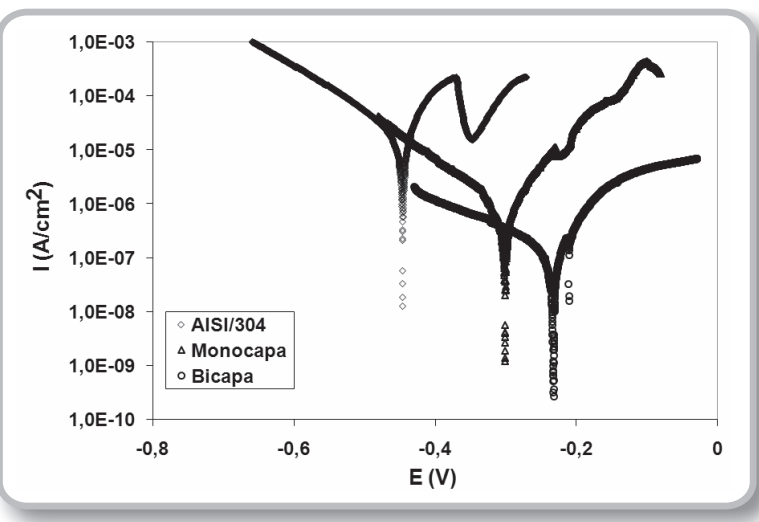

(a)

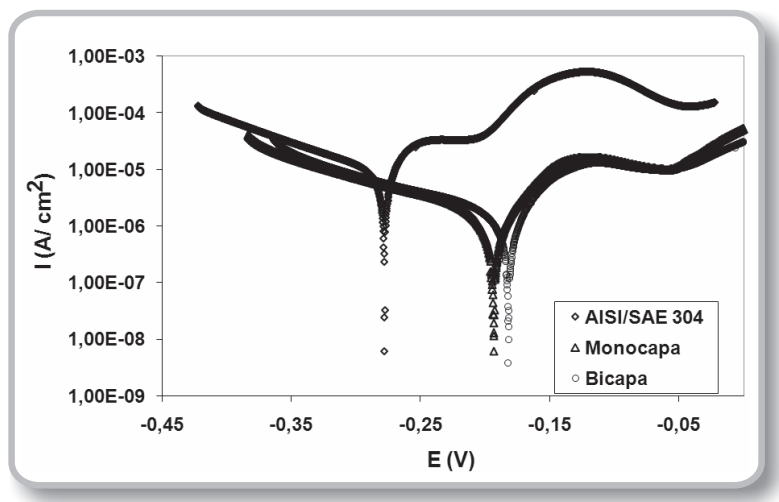

(b) 
En la tabla 2, 3 y 4 se reportan los valores del potencial, corriente y velocidad de corrosión para el sustrato y los recubrimientos en monocapa y bicapa según el proceso de sinterizado vía plasma y convencional.

Un factor importante a tener en cuenta cuandosedesarrollanestudioselectroquímicos, son los valores de velocidad de corrosión. Una óptima película protectora a la corrosión es aquella que ofrece valores muy bajos de velocidad de corrosión. Esto permite afirmar que la película actúa como una barrera eficiente ante el ataque de los iones presente en la solución.

Tabla 2. Valores del potencial de corrosión, para el sustrato y los recubrimientos en monocapa y bicapa sinterizados vía plasma y convencional.

\begin{tabular}{|c|c|c|c|}
\hline Vía de & \multicolumn{3}{|c|}{ - Potencial de corrosión (mV) } \\
\cline { 2 - 4 } sinterizado & Monocapa & Bicapa & AISI/304 \\
\hline Plasma & 301 & 201 & \multirow{2}{*}{446} \\
\hline Convencional & 194 & 182 & \\
\hline
\end{tabular}

Tabla 3. Valores de corrientes de corrosión, para el sustrato y los recubrimientos en monocapa y bicapa sinterizados vía plasma y convencional.

\begin{tabular}{|c|c|c|c|}
\hline Vía de & \multicolumn{3}{|c|}{ Corriente de corrosión $(\boldsymbol{\mu} \boldsymbol{A})$} \\
\cline { 2 - 4 } sinterizado & Monocapa & Bicapa & AISI/304 \\
\hline Plasma & 1,12 & 0,17 & \multirow{2}{*}{17,90} \\
\hline Convencional & 3,27 & 2,02 & \\
\hline
\end{tabular}

Tabla 4. Valores de velocidad de corrosión, para el sustrato y los recubrimientos en monocapa y bicapa sinterizados vía plasma y convencional.

\begin{tabular}{|c|c|c|c|}
\hline \multirow{2}{*}{ Vía de } & \multicolumn{3}{|c|}{ Velocidad de corrosión (mpy) } \\
\cline { 2 - 3 } sinterizado & Monocapa & Bicapa & AISI/304 \\
\hline Plasma & $916,7 \times 10^{-3}$ & $122 \times 10^{-3}$ & \multirow{2}{*}{14,58} \\
\hline Convencional & 16,45 & 7,53 & \\
\hline
\end{tabular}

De los resultados anteriores, se aprecia que el proceso de sinterización vía plasma es más eficiente que el convencional. Esta eficiencia radica en que para el proceso convencional el calentamiento del sustrato es volumétrico, mientras que para proceso vía plasma el calentamiento es superficial.

Cuando se densifican películas cerámicas vía convencional sobre sustratos de acero AISI/304 es muy importante tener en cuenta la inestabilidad del sustrato a temperaturas mayores de $500^{\circ} \mathrm{C}$, debido a la precipitación, en las fronteras de grano, de carburos de cromo. Por el contrario, la sinterización vía plasma ofrece un tratamiento térmico superficial a mayores temperaturas; esto favorece la sinterización de los recubrimientos permitiendo un óptimo sellado de los mismos y lo más importante, no produce sensibilización del sustrato.

También se comprobó que un sistema rico en titanio $[10,11]$ en la composición de películas cerámicas, permite obtener una alto grado de efectividad que evita el ataque de los iones cloruro al sustrato AISI/304, efecto que tiene mayor incidencia al aumentar el número de capas aplicadas.

Los resultados obtenidos, se relacionan con la caracterización superficial, buenos recubrimientos anticorrosivos presentan buena homogeneidad y pocas imperfecciones.

\section{Conclusiones}

Se concluye que la eficiencia del comportamiento anticorrosivo de las películas se da por un factor importante, el número de capas aplicadas al sustrato. A medida que aumenta el número de capas, las películas posteriores homogenizan la superficie de las primeras capas mediante el sellamiento de poros y grietas.

Del estudio experimental se comprobó la viabilidad de desarrollar el proceso de sinterización vía plasma de recubrimiento 
en monocapa y bicapa del sistema ternario $\mathrm{SiO} 2 \mathrm{TiO} 2 \mathrm{ZrO} 2$ en la concentración $\mathrm{Si} / \mathrm{Ti} /$ $\mathrm{Zr}$ : 10/70/20 aplicados sobre el sustrato de acero AISI/304 por la técnica de deposición por inmersión (Dip-coating). Esta viabilidad se refleja en los resultados encontrados, en especial los referentes a corrientes de corrosión.

Los recubrimientos en bicapa sinterizados vía plasma muestran menor susceptibilidad a la corrosión según análisis electroquímico; las velocidades de corrosión disminuyeron en tres órdenes de magnitud con respecto al sustrato de acero AISI/304.

\section{Agradecimientos}

Esta investigación fue financiada por el Instituto Colombiano para el Desarrollo de la Ciencia y la Tecnología "Francisco José de Caldas"-COLCIENCIAS, en el marco de desarrollo del proyecto: "Recubrimientos cerámicos e híbridos funcionales con resistencia a la corrosión y con propiedades ópticas”, código 1109-06-17621.

\section{Bibliografía}

1. C. J. Brinker, G. W. Scherer, "Sol-Gel Science: the physics and chemistry of solgel processing"; 1990, Academic Press, Inc.

2. L. L. Hech, "Science of Ceramic Chemical Processing”; 1986, John Wiley \& Sons, Inc.

3. L. Klein, "Sol-gel technology for thin films, fibers, performs, electronics and specialty shapes, Noyes"; 1988. Park Ridge.

4. J. Camargo, "Estudio del proceso de sinterización por plasma de recubrimientos cerámicos tipo $\mathrm{SiO} 2 \mathrm{TiO} 2 \mathrm{ZrO} 2$ obtenidos por el método sol-gel”, Monografía,
Universidad Pedagógica y Tecnológica de Colombia. (2007)

5. J. Livage, "Better Ceramic Through chemistry"; Material Research Society, Vol. 73 (1986), p 717-724.

6. W. C. La Course, S. Kirn, "Science of Ceramic Chemical Processing"; 1986, John Wiley and Sons, Inc.

7. F. Mansfeld, "Evaluation of corrosive degradation in coated steel using alternating current impedance spectroscopy"; Corros. Sci., Vol. 52 (6) (1996), p. 417.

8. M. Stratmann, K. Hoffmann, "Proceedings of the Ninth European Congress of Corrosion", Utrecht, The Netherlands, p. CO-70 (1989).

9. L.F. Vesga, E Vera, J.H. Panqueva, "Use of the Electrochemical Impedance Spectroscopy to evaluate the performance of a primer applied under different surface preparation method"; Progress in Organic Coatings, Vol. 39 (2000), p. 61-65.

10.J. Bautista, "Producción y caracterización de películas cerámicas $\mathrm{SiO} 2 \mathrm{TiO} 2 \mathrm{ZrO} 2$ sintetizadas por el método Sol-gel para aplicaciones anticorrosivas", Tesis de Maestría, Universidad Pedagógica y Tecnológica de Colombia. (2006).

11.J. Bautista, "Conformación de películas delgadas tipo sol-gel para aplicaciones anticorrosivas"; Colección Investigación № 20. UPTC, Tunja- Colombia, 2008. 